\title{
BMJ Open Is hypercapnia associated with poor prognosis in chronic obstructive pulmonary disease? A long-term follow-up cohort study
}

\author{
Hui Yang, ${ }^{1,2}$ Pingchao Xiang, ${ }^{2}$ Erming Zhang, ${ }^{2}$ Weian Guo, ${ }^{2}$ Yanwei Shi, ${ }^{2}$ \\ Shuo Zhang, ${ }^{2}$ Zhaohui Tong $^{1}$
}

To cite: Yang $\mathrm{H}$, Xiang $\mathrm{P}$, Zhang $\mathrm{E}$, et al. Is hypercapnia associated with poor prognosis in chronic obstructive pulmonary disease? A long-term follow-up cohort study. BMJ Open 2015:5:e008909 doi:10.1136/bmjopen-2015008909

- Prepublication history for this paper is available online. To view these files please visit the journal online (http://dx.doi.org/10.1136/ bmjopen-2015-008909).

Received 27 May 2015 Revised 28 October 2015 Accepted 29 October 2015

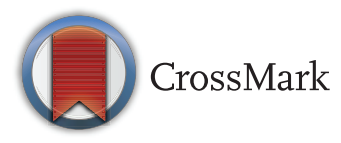

${ }^{1}$ Department of Respiratory and Critical Care Medicine, Beijing Institute of Respiratory Medicine, Beijing Chao-Yang Hospital Beijing, Capital Medical University, Beijing, China

${ }^{2}$ Department of Respiratory and Critical Care Medicine, Shou-Gang Hospital Affiliated to Peking University, Beijing, China

Correspondence to Dr Zhaohui Tong; chaohuitongsci@163.com

\section{ABSTRACT}

Objectives: To assess whether hypercapnia may predict the prognosis in chronic obstructive pulmonary disease (COPD).

Design: Prospective cohort study comparing the survival of patients with COPD and normocapnia to those with chronic hypercapnia.

Setting: Patients with consecutive COPD were enrolled between 1 May 1993 and 31 October 2006 at two medical centres. Follow-up was censored on 31 October 2011.

Participants: A total of 275 patients with stable COPD and aged 40-85 years were enrolled. Diagnosis of hypercapnia was confirmed by blood gas analysis. Patients with near-terminal illness or comorbidities that affect $\mathrm{PaCO}_{2}$ (obstructive sleep apnoea, obesity-related hypoventilation, or neuromuscular disease) were excluded. The outcome of 98 patients with normocapnia and 177 with chronic hypercapnia was analysed.

Outcome measures: Overall survival.

Results: Median survival was longer in patients with normocapnia than in those with hypercapnia (6.5 vs 5.0 years, $p=0.016)$. Multivariate COX regression analysis indicated that age $(\mathrm{HR}=1.043,95 \% \mathrm{Cl} 1.012$ to 1.076), Charlson Index, which is a measure of comorbidity ( $\mathrm{HR}=1.172,95 \% \mathrm{Cl} 1.067$ to 1.288 ), use of medication ( $\mathrm{HR}=0.565,95 \% \mathrm{Cl} 0.379$ to 0.842 ), body mass index (BMI) (HR=0.922, $95 \% \mathrm{Cl} 0.883$ to 0.963 ), $\mathrm{PaCO}_{2}$ (HR=1.026, 95\% Cl 1.011 to 1.042), Cor pulmonale (HR=2.164, 95\% Cl 1.557 to 3.006$)$, noninvasive positive-pressure ventilation (NPPV) (HR=0.615, 95\% Cl 0.429 to 0.881 ) and per cent of forced expiratory volume in $1 \mathrm{~s}$ (FEV1\%) (HR=0.979, $95 \% \mathrm{Cl} 0.967$ to 0.991 ), were independent risk factors for mortality.

Conclusions: Increased age, Charlson Index, chronic hypercapnia and Cor pulmonale, and decreased FEV $1 \%$, use of medication, BMI and NPPV, were associated with a poor prognosis in patients with COPD.

\section{INTRODUCTION}

Chronic obstructive pulmonary disease (COPD) should no longer be regarded as a

\section{Strengths and limitations of this study}

- This study was followed up for more than 10 years and has important clinical significance for the prognosis of COPD in China.

- Previous studies on the prognosis of COPD with hypercapnia demonstrated a lack of clinical data.

- There was a substantial loss of patients to follow-up during the dynamic monitoring of the changes in PaCO2 and FEV1.

simple airflow obstructive disease, but should instead be considered a condition that comprises several phenotypes. ${ }^{1-3}$ The traditional forced expiratory volume in $1 \mathrm{~s} \mathrm{(FEV1)} \mathrm{meas-}$ urement is insensitive to emphysema severity in COPD, and patients with similar FEV1 values may exhibit very different underlying pathologies. Spirometric assessment alone seems insufficient for the characterisation of COPD, and there may be a need for a more detailed understanding of the conditions that are included in COPD.

Chronic hypercapnia occurs in cases of impaired respiratory control combined with an impairment of $\mathrm{HCO}_{3}^{-}$handling by the kidneys, resulting in arterial carbon dioxide tension $\left(\mathrm{PaCO}_{2}\right)$ constantly $>45 \mathrm{~mm} \mathrm{Hg.}{ }^{4} 5$ Chronic hypercapnia occurs in some patients with COPD, but not in all. ${ }^{1}{ }^{4}$ Some patients hospitalised for acute hypercapnia eventually reverse to normocapnia after recovery. Some studies reported that hypercapnia was a poor prognostic indicator for patients with COPD, and that survival in patients with hypercapnia with COPD was shorter than in normocapnia and reversible patients with hypercapnia with COPD, whereas the survival time of patients with reversible hypercapnia was similar to that of patients with normocapnia, ${ }^{4}$ but these observations 
are controversial ${ }^{6} 7$ and a number of factors are involved in the survival of patients with COPD. ${ }^{8} 9$ Non-invasive positive-pressure ventilation (NPPV) may improve the prognosis of patients with COPD and hypercapnia. ${ }^{10}$

A limitation of these studies is the relatively short follow-up. The objective of this study was to evaluate the prognostic factors of patients with COPD in a long-term follow-up setting, and to investigate the differences between normocapnia and hypercapnia in COPD.

\section{MATERIALS AND METHODS}

\section{Study design and patients}

This was a prospective cohort survival study designed to compare patients with COPD with normocapnia to those with chronic hypercapnia. Patients with consecutive COPD were enrolled at two medical centres between 1 May 1993 and 31 October 2006. COPD was diagnosed according to the standard American Thoracic Society (ATS) criteria. ${ }^{1}$ The patients were admitted due to acute exacerbation of COPD (AECOPD), and discharged from the hospital after improvements of disease conditions after treatments. The patients were followed up at 6 weeks, and those with stable illness (no coughing, no phlegm, no increasing asthma symptoms and no change in drug use) were considered as stable and included in this study.

The inclusion criteria were: (1) stable COPD at entry (ie, no coughing, no phlegm, no increasing asthma symptoms and no change in drug use for at least 6 weeks); (2) age 40-85 years; and (3) blood gas analysis performed 6 weeks after discharge confirming the diagnosis. The exclusion criteria were: (1) any near-terminal illness ( $<1$ month of life expectancy) or (2) comorbidities that could affect $\mathrm{PaCO}_{2}$ (obstructive sleep apnoea, obesity-related hypoventilation or neuromuscular disease). The study was approved by the ethics review boards at the two participating centres, and informed written consent was obtained from each patient.

\section{Data collection}

Although the patients were prospectively recruited and followed up, some standard clinical data were retrieved from the medical charts (such as clinical symptom, signs, use of medication, long-term oxygen therapy (LTOT), NPPV at home or at hospital and comorbidities). Data that were prospectively collected were also crosschecked with the medical charts whenever possible. During their initial clinical examinations, patient demographics were recorded including sex, age, height, weight and smoking history. Body mass index (BMI) was calculated $\left(\mathrm{kg} / \mathrm{m}^{2}\right)$.

\section{Outcome assessment}

Arterial blood gases were measured at recruitment and every 2 years, using an ABL700 blood gas analyser (Radiometer, Copenhagen, Denmark) while the patients were breathing room air. Measurements were performed after $5-10 \mathrm{~min}$ of seated rest. For those requiring it, oxygen was stopped for $20 \mathrm{~min}$ before measurement. Normocapnia was defined as blood gas $\mathrm{PaCO}_{2}$ $\leq 45 \mathrm{~mm} \mathrm{Hg}$. Chronic hypercapnia was defined as $\mathrm{PaCO}_{2}$ $>45.0 \mathrm{~mm} \mathrm{Hg}$ recorded twice at an interval of at least 6 weeks.

Lung function was assessed by spirometry (HP 47-804; Hewlett-Packard, Waltham, Massachusetts, USA) at recruitment and every 2 years thereafter, according to the ATS guidelines. ${ }^{1}$ Lung function was determined by the grades of airflow obstruction according to standard ATS criteria: ${ }^{1}$ grade 1: FEV1/forced vital capacity (FVC) $<70 \%$, FEV1\%, predicted $\geq 80 \%$; grade 2: FEV1/ FVC $<70 \%, \quad 50 \% \leq \mathrm{FEV} 1 \%$, predicted $<80 \%$; grade 3: FEV1 $/$ FVC $<70 \%, 30 \% \leq \mathrm{FEV} 1 \%$, predicted $<50 \%$; and grade 4: FEV1/FVC $<70 \%$, FEV1\%, predicted $<30 \%$. AECOPD was defined as 'an acute worsening of the patient's condition from a stable state of COPD that is sustained and may warrant the patient to seek additional treatment'. ${ }^{11}$ ECG, chest radiography, chest CT and/or echocardiography were measured at recruitment. The diagnosis of chronic Cor pulmonale was based on both clinical evidences (physical evidence of peripheral oedema, increased intensity of pulmonary component of the second heart sound or tricuspid regurgitation murmur), echocardiography data, chest radiography demonstrating an increased width of the right descending pulmonary artery or right ventricle (RV) enlargement, and ECG demonstrating P-pulmonale, right axis deviation or RV hypertrophy. ${ }^{12}$ Echocardiography (Vivid Eq, GE UItrasound System, Horten, Norway) was used to evaluate chronic Cor pulmonale. ${ }^{13} 14$

Significant comorbidities were recorded and quantified according to the well-established Charlson Index. ${ }^{15}$ Comorbidities are scored 1 (myocardial infarct, congestive heart failure, peripheral vascular disease, dementia, cerebrovascular disease, chronic lung disease, ulcer, chronic liver disease, diabetes), 2 (hemiplegia, moderate or severe kidney disease, diabetes with end organ damage, tumour, leucaemia, lymphoma), 3 (moderate or severe liver disease) or 6 (malignant tumour, metastasis, AIDS) points; scores are summed to provide a score predicting mortality. ${ }^{15}$ The definition of comorbidity was based on the presence of one or more distinct disorders (or diseases) in addition to COPD, regardless of whether or not the comorbidities were directly related to COPD. ${ }^{16}$

\section{Follow-up}

Follow-up was censored on 31 October 2011 or at the time of death. Following the recruitment visit, patients were followed up every 6 months by telephone or through their medical records, including the need for LTOT, use of medication, newly discovered comorbidities, and the use of NPPV at home and at the hospital. The main causes of death were recorded for each patient. Lung function and arterial blood gases were assessed every 2 years. 


\section{Statistical analysis}

Continuous variables are presented as means \pm SDs or as median and range between the 5th and 95th centiles. For continuous variables, groups were compared using independent sample $\mathrm{t}$ tests or Mann-Whitney U-tests, as appropriate. $\chi^{2}$ Tests were used for categorical variables. Survival analysis was performed using Kaplan-Meier analysis with the log-rank test. The outcome was death from any cause (overall survival). Multivariate Cox regression analysis was used to identify the independent predictors of survival. All analyses were performed using SPSS V.17.0 (IBM, Armonk, New York, USA). The $\mathrm{p}<0.05$ was considered to be statistically significant.

\section{RESULTS}

\section{Patient characteristics}

Patients were admitted due to AECOPD, and 157 patients with normocapnia and 253 patients with hypercapnia (including those with chronic hypercapnia) were discharged from the hospital due to improved disease conditions after treatment. In the normocapnia group, 6-week follow-up revealed AECOPD in 21 patients, two were lost to follow-up and 36 refused to undergo the 6 -week blood gas analysis; 98 patients with normocapnia were included. Among 253 patients with hypercapnia, 56 patients had AECOPD, 5 were lost to follow-up, 1 died and 14 refused the 6-week blood gas examination; 177 patients with hypercapnia were included (figure 1).

As shown in table 1, patients with normocapnia were older $(p=0.013)$ and had lower rates of medication use $(\mathrm{p}=0.006)$ compared to those with hypercapnia. Patients with normocapnia showed elevated arterial $\mathrm{pH}, \mathrm{PaO}_{2}$, Charlson Index and rate of comorbidities. NPPV use and Cor pulmonale were higher in patients with hypercapnia.

\section{Changes in arterial blood gases and lung function}

Changes in arterial blood gases and lung function in the two groups were assessed every 2 years during follow-up (figure 2). Thirty-nine patients with normocapnia and 72 with hypercapnia were dynamically monitored for arterial blood gas and lung function. The mean fluctuation range of $\mathrm{PaCO}_{2}$ was $11.9 \mathrm{~mm} \mathrm{Hg}$ in patients with normocapnia and $22 \mathrm{~mm} \mathrm{Hg}$ in patients with hypercapnia. Among patients with normocapnia, carbohemia was kept relatively stable in 24 patients $(61.54 \%)$, reversible hypercapnia occurred in 13 patients $(33.33 \%, 13 / 39)$ and 2 patients $(5.12 \%, 2 / 39)$ developed chronic hypercapnia. The dynamic curve showed a small fluctuation of $\mathrm{PaCO}_{2}$ in patients with normocapnia, and a large fluctuation and a progressive increase of $\mathrm{PaCO}_{2}$ in patients with hypercapnia. The FEV1 in patients with both normocapnia and hypercapnia exhibited a progressive reduction, while the reduction in normocapnia was greater than that in hypercapnia (annualised rate; 0.86 \pm 0.35 vs $0.62 \pm 0.22 \mathrm{~L}, \mathrm{p}<0.001)$.

\section{Survival analysis and multivariate COX regression analysis}

Follow-up was 5.6 \pm 3.3 (range 0.3-14.6) years in patients with normocapnia, and 5.0 3.5 (range 0.3-14.0) years in those with hypercapnia. Among patients with normocapnia, 52 of $98(53.06 \%)$ died and $8(9.18 \%)$ dropped out during follow-up. Among patients with hypercapnia, 127 of $177(71.75 \%)$ died and $4(2.30 \%)$ dropped out. Median survival was longer in patients with normocapnia compared to those with hypercapnia (6.5 vs 5.0 years, $\mathrm{p}=0.016$ ) (figure 3 ).

Age, sex, smoking, BMI, LTOT, use of medication, $\mathrm{pH}$, $\mathrm{PaCO}_{2}, \mathrm{PaO}_{2}, \mathrm{FEV} 1$, FVC, FEV1\%, Cor pulmonale, Charlson Index and NPPV were included in the multivariate Cox regression analysis. The results showed that age $(\mathrm{HR}=1.043,95 \%$ CI 1.012 to $1.076, \mathrm{p}=0.007)$, Charlson Index (HR=1.172, 95\% CI 1.067 to 1.288 , $\mathrm{p}=0.001)$, use of medication $(\mathrm{HR}=0.565$, 95\% CI 0.379 to $0.842, \mathrm{p}=0.005$ ), BMI ( $\mathrm{HR}=0.922,95 \%$ CI 0.883 to $0.963, \mathrm{p}<0.001), \mathrm{PaCO}_{2}(\mathrm{HR}=1.026,95 \%$ CI 1.011 to 1.042, $\mathrm{p}=0.001)$, Cor pulmonale $(\mathrm{HR}=2.164,95 \%$ CI

Figure 1 Study flow chart (COPD, chronic obstructive pulmonary disease).

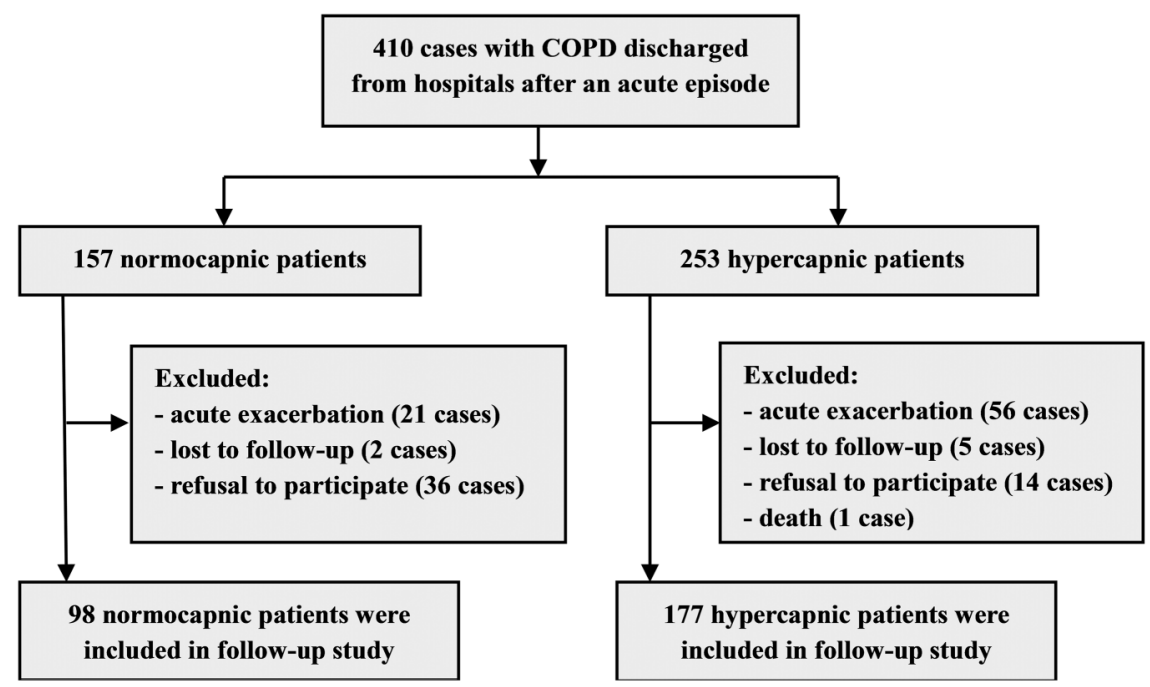


Table 1 Baseline characteristics of the patients in the normocapnic and chronic hypercapnic groups

\begin{tabular}{|c|c|c|c|}
\hline Parameters & Normocapnia $(n=98)$ & Chronic hypercapnia $(\mathrm{n}=177)$ & p Value \\
\hline Age, year & $73.70 \pm 6.91$ & $71.49 \pm 7.09$ & 0.013 \\
\hline Male, n (\%) & $70(71.43)$ & $128(72.32)$ & 0.955 \\
\hline Smoking, n (\%) & $60(61.22)$ & $97(54.80)$ & 0.303 \\
\hline $\mathrm{BMI}, \mathrm{kg} / \mathrm{m}^{2}$ & $22.92 \pm 3.10$ & $23.78 \pm 3.89$ & 0.052 \\
\hline LTOT, n (\%) & $9(9.18)$ & $18(10.17)$ & 0.255 \\
\hline Use of medication, $\mathrm{n}(\%)$ & $18(18.37)$ & $60(33.90)$ & 0.006 \\
\hline Theophylline & $17(17.35)$ & 39 (22.03) & 0.355 \\
\hline Fluticasone/salmeterol & $1(1.02)$ & $24(13.56)$ & $<0.001$ \\
\hline Tiotropium bromide & $0(0)$ & $4(2.26)$ & $<0.001$ \\
\hline FVC, L & $1.71 \pm 0.55$ & $1.24 \pm 0.41$ & $<0.001$ \\
\hline FEV1, L & $0.96 \pm 0.37$ & $0.62 \pm 0.24$ & $<0.001$ \\
\hline $\mathrm{pH}$ & $7.42 \pm 0.03$ & $7.38 \pm 0.04$ & $<0.001$ \\
\hline $\mathrm{PaO}_{2}, \mathrm{~mm} \mathrm{Hg}$ & $69.26 \pm 7.17$ & $63.23 \pm 8.13$ & $<0.001$ \\
\hline $\mathrm{PaCO}_{2}, \mathrm{~mm} \mathrm{Hg}$ & $39.20 \pm 4.70$ & $57.39 \pm 7.82$ & $<0.001$ \\
\hline Cor pulmonale, $\mathrm{n}(\%)$ & $24(24.39)$ & $117(66.10)$ & $<0.001$ \\
\hline Charlson Index & $6.071 \pm 1.863$ & $4.791 \pm 1.754$ & $<0.001$ \\
\hline \multicolumn{4}{|l|}{ NPPV, n (\%) } \\
\hline NPPV at home & $0(0)$ & $6(0.4)$ & $<0.001$ \\
\hline NPPV in hospital & $8(8.2)$ & $63(35.6)$ & $<0.001$ \\
\hline Comorbidities & $89(90.82)$ & $110(62.15)$ & $<0.001$ \\
\hline Coronary disease & $42(42.9)$ & $37(20.9)$ & $<0.001$ \\
\hline Hypertension & $40(40.8)$ & $56(31.6)$ & 0.126 \\
\hline Cerebrovascular & $44(44.9)$ & $29(16.4)$ & $<0.001$ \\
\hline Diabetes & $20(20.4)$ & $11(6.2)$ & $<0.001$ \\
\hline Lung cancer & $5(5.1)$ & $13(7.3)$ & 0.471 \\
\hline Other tumours & $5(5.1)$ & $2(1.1)$ & 0.101 \\
\hline Pulmonary embolism & $4(4.1)$ & $2(1.1)$ & 0.191 \\
\hline
\end{tabular}

BMI, body mass index; FEV1, forced expiratory volume in $1 \mathrm{~s}$; FVC, forced vital capacity; LTOT, long-term oxygen therapy; NPPV, non-invasive positive-pressure ventilation; $\mathrm{PaO}_{2}$, arterial oxygen tension; $\mathrm{PaCO}_{2}$, arterial carbon dioxide tension.

1.557 to $3.006, \mathrm{p}<0.001)$, NPPV $(\mathrm{HR}=0.615,95 \%$ CI 0.429 to $0.881, \mathrm{p}=0.008)$ and $\mathrm{FEV} 1 \% \quad(\mathrm{HR}=0.979,95 \%$ CI 0.967 to $0.991, \mathrm{p}=0.001)$, were independent risk factors of overall survival (table 2).

\section{Comorbidities and causes of death}

As aforementioned, the proportion of patients with comorbidities among those with normocapnia was higher compared to those with hypercapnia $(90.8 \%$ vs $62.2 \%$, respectively, $\mathrm{p}<0.001)$. The three most common comorbidities in both groups were hypertension, coronary artery disease and cerebrovascular disease (table 1).

Among 275 patients, 179 (65.09\%) died during follow-up, and the three most common causes of death were respiratory failure $(90 / 179,50.28 \%)$, pneumonia $(26 / 179,14.53 \%)$ and cerebrovascular disease $(11 / 179$, $6.15 \%)$. Among patients with normocapnia, 52 of 98 $(53.06 \%)$ died during the study, and the main causes of death were pneumonia $(11 / 52,21.15 \%)$, cerebrovascular disease $(6 / 52,11.54 \%)$, cardiogenic diseases (coronary artery disease, heart failure) $(6 / 52,11.54 \%)$, lung cancer $(6 / 52,11.54 \%)$ and respiratory failure $(6 / 52$, $11.54 \%$ ). Among patients with hypercapnia, 127 of 177 $(71.75 \%)$ died, and the main causes of death were respiratory failure $(84 / 127,66.14 \%)$, pneumonia (15/ $127,11.81 \%)$ and lung cancer $(7 / 127,5.51 \%)$. The incidence of death being due to respiratory failure was significantly higher in patients with hypercapnia patients than in patients with normocapnia (OR, 14.977; 95\% CI 5.929 to 37.835 ), whereas no significant differences between groups were observed for the other causes of death (table 3).

The median survival of patients with hypercapnia with Cor pulmonale was shorter compared to that of patients with normocapnia with Cor pulmonale (4.8 vs 6.0 years). The 3-year and 6-year cumulative survival rates in patients with normocapnia were $72 \%$ and $20 \%$, whereas these rates were $50 \%$ and $17 \%$ in patients with hypercapnia, respectively, but the differences were not significant $(\mathrm{p}>0.05)$.

\section{DISCUSSION}

An important finding of the present study is that patients with COPD with hypercapnia had a poor prognosis compared to those with normocapnia. To determine whether patients with normocapnia subsequently develop chronic hypercapnia remains an area of controversy. The core of this question is to determine if normocapnia and hypercapnia are two stages of the same disease, or whether they are two different entities. Costello et at observed that only $24 \%$ of patients with 

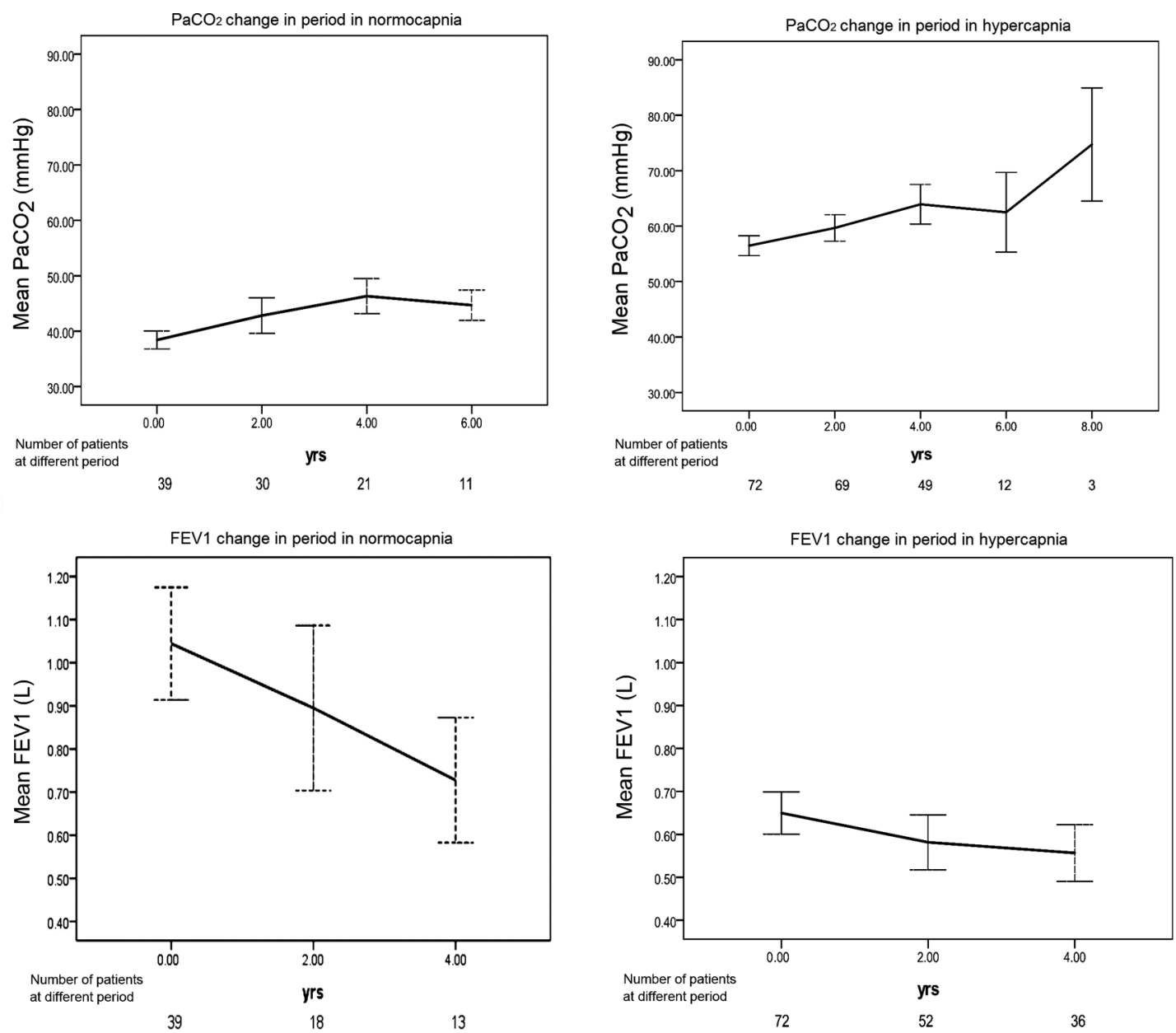

Figure 2 Comparison of the changes in time of arterial carbon dioxide tension $\left(\mathrm{PaCO}_{2}\right)$ and forced expiratory volume in $1 \mathrm{~s}$ (FEV1) between patients with normocapnia and hypercapnia.

reversible hypercapnia developed chronic hypercapnia during follow-up. In this study, among patients classified as normocapnic at recruitment, $5.12 \%$ of patients with normocapnia $(13.3 \%$ of patients with reversible hypercapnia) developed chronic hypercapnia during longterm follow-up. In general, the results of this study are consistent with those of Costello et al. ${ }^{4}$ This could imply that COPD with normocapnia may not simply be an early stage of COPD with hypercapnia, but may represent a distinct COPD phenotype.

Previous studies showed that about $25 \%$ of patients presenting AECOPD were hypercapnic, ${ }^{1} 1117$ while this frequency was $62 \%$ in the present study. This may be due to this specific population of Chinese patients having different lifestyle and treatment characteristics than those of Western populations. The high frequency of smokers could confound the results. Among the 275 patients, 154 had lung function grade 4 and 78 had grade 3 . Therefore, $84 \%$ of the patients had a severe or highly severe condition. This could explain the high rate of hypercapnia. Additional studies with larger numbers of patients are necessary to address this issue adequately.

A previous study showed a higher (but not statistically significant) mortality rate in patients with chronic

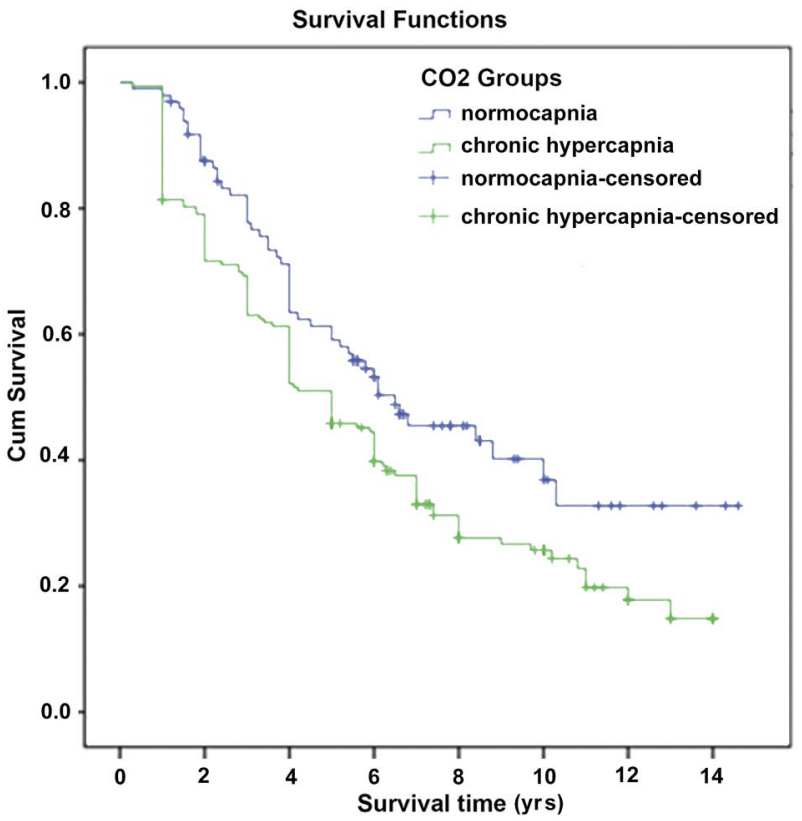

Figure 3 Comparison of survival curves in all patients between groups with normocapnia and chronic hypercapnia $(p=0.016)$. 
Table 2 Multivariate COX regression analysis for survival

\begin{tabular}{lllr}
\hline Items & HR & 95\% Cl & p Value \\
\hline Age, years & 1.043 & 1.012 to 1.076 & 0.007 \\
BMI, kg/m & 0.922 & 0.883 to 0.963 & $<0.001$ \\
Charlson Index $^{2}$ & 1.172 & 1.067 to 1.288 & 0.001 \\
PaCO $_{2}, \mathrm{~mm} \mathrm{Hg}$ & 1.026 & 1.011 to 1.042 & 0.001 \\
FEV1\% $_{\text {Cor pulmonale }}$ & 0.979 & 0.967 to 0.991 & 0.001 \\
NPPV & 2.164 & 1.557 to 3.006 & $<0.001$ \\
Use of medication & 0.615 & 0.429 to 0.881 & 0.008 \\
\hline
\end{tabular}

BMI, body mass index; FEV1, forced expiratory volume in $1 \mathrm{~s}$; FVC, forced vital capacity; LTOT, long-term oxygen therapy; NPPV, non-invasive positive-pressure ventilation; $\mathrm{PaCO}_{2}$, arterial carbon dioxide tension.

hypercapnia compared to normocapnia. ${ }^{4}$ Another study with a 10-year follow-up failed to observe a difference in survival between patients with hypercapnia and reversible hypercapnia. ${ }^{7}$ Furthermore, in support of Costello et $a l,{ }^{4}$ survival was longer in patients with normocapnia compared to those with hypercapnia, which supports the present study.

Zielinski et $_{a l} \mathrm{l}^{19}$ showed that respiratory failure was the leading cause of death in patients with COPD; other causes were cardiovascular diseases, pulmonary infection, pulmonary embolism, lung cancer and other cancers. The SUPPORT trial showed that comorbidities played a major role, even in 'COPD-specific' deaths among patients with very advanced COPD. ${ }^{20}$ In the present study, the three most common causes of death were respiratory failure $(50 \%)$, pneumonia $(15 \%)$ and lung cancer $(7 \%)$, consistent with the earlier findings. In addition, among patients with chronic hypercapnia, respiratory failure was the main cause of death. Some studies have suggested that the main reason for developing comorbidities concomitantly with COPD was the systemic inflammation state associated with COPD,${ }^{21}$ and that smoking ${ }^{22}$ and ageing ${ }^{23}$ also play key roles in this process. A large study from the UK showed that patients with COPD had a significantly increased risk of comorbidities and other medical events, ${ }^{24}$ particularly comorbidities related to cardiovascular-related, bonerelated and other smoking-related conditions. A study revealed that patients with COPD had a higher prevalence of cardiovascular diseases, local malignant neoplasm and neurological diseases other than stroke with hemiplegia, ulcer and gastritis. ${ }^{25}$ The present study showed that the most common comorbidities in both groups were hypertension, coronary artery disease and cerebrovascular disease. In addition, the incidences of coronary artery diseases, cerebrovascular diseases and diabetes mellitus were higher in patients with normocapnia compared with those in patients with hypercapnia, further demonstrating a greater incidence of comorbidities in patients with normocapnia. Indeed, some studies have suggested a protective effect of hypercapnia against ischaemia-reperfusion injuries. ${ }^{26}{ }^{27}$ However, the higher use of medication in the hypercapnia group may confound these results, despite the fact that a higher proportion of smoking was observed among patients with hypercapnia. Further studies with larger samples are necessary to address these issues adequately.

Cor pulmonale is defined as structural changes and functional deterioration of the RV resulting from pulmonary hypertension, which is associated with various lung, upper airway and chest wall diseases. ${ }^{28}$ Cor pulmonale, a common type of heart disease as a result of its close association with COPD, has emerged as a leading cause of disability and death. ${ }^{29}$ In the present study, patients with hypercapnia had low $\mathrm{PaO}_{2}$ and a higher frequency of Cor pulmonale. Analysis revealed that, for patients with Cor pulmonale, the survival of patients with hypercapnia was shorter compared to that in normocapnia. However, the definition used for $\mathrm{PaO}_{2}$ might influence these results and prevent direct comparisons between studies. LTOT is one of the main treatments for patients with stage IV COPD and is associated with improved survival. ${ }^{17}{ }^{30}$ However, its use in a domestic environment is far from ideal. ${ }^{31}$ Studies have shown that compliance in patients receiving LTOT is low. ${ }^{32-34}$ In this study, only $9.2 \%$ of the patients with normocapnia and $10.2 \%$ of the patients with hypercapnia received LTOT. Multivariate analysis revealed that LTOT was not a prognostic factor for patients with COPD, but the high proportion of smokers might influence this result.

NPPV therapy is an established strategy for treating COPD and has been shown to decrease mortality, acute exacerbations and requirement for intubation. ${ }^{17} 3035 \mathrm{In}$

Table 3 The main causes of death between normocapnia and chronic hypercapnia in patients with chronic obstructive pulmonary disease

\begin{tabular}{|c|c|c|c|c|c|}
\hline \multirow[b]{2}{*}{ Main causes of death } & \multicolumn{2}{|c|}{$\begin{array}{l}\text { Normocapnia (death) } \\
(n=52)\end{array}$} & \multicolumn{2}{|c|}{$\begin{array}{l}\text { Hypercapnia (death) } \\
(\mathrm{n}=127)\end{array}$} & \multirow[b]{2}{*}{ p Value } \\
\hline & $\mathbf{N}$ & Per cent & $\mathbf{N}$ & Per cent & \\
\hline Pneumonia & 11 & 21.15 & 15 & 11.81 & 0.107 \\
\hline Cerebrovascular diseases & 6 & 11.54 & 5 & 3.94 & 0.055 \\
\hline Cardiac diseases & 6 & 11.54 & 5 & 3.94 & 0.055 \\
\hline Lung cancer & 6 & 11.54 & 7 & 5.51 & 0.158 \\
\hline Respiratory failure* & 6 & 11.54 & 84 & 66.14 & $<0.0001$ \\
\hline
\end{tabular}


this study, NPPV was also associated with increased median survival, confirming its protective effect in patients with hypercapnia with COPD, but not in patients with normocapnia, which might be because of the small proportion of NPPV users in this study. COX regression analysis showed that NPPV was a beneficial factor for the prognosis of chronic hypercapnia.

In addition, the Cox regression analysis revealed that drug therapy (notably fluticasone propionate and salmeterol) was an independent factor for the prognosis of patients with COPD, mainly for patients with hypercapnia, because patients with normocapnia only used fluticasone propionate and salmeterol inhalation. Further studies are required to investigate whether use of fluticasone propionate and salmeterol achieves an efficacy in normocapnia similar to that of hypercapnia. At the same time, this study suggests that increasing age, lower FEV1 and lower BMI increased the risk of death. Taken together, these results suggest that NPPV and lung function are important factors in patients with COPD.

This study is not without its limitations. First, there was a substantial loss of patients to follow-up during the dynamic monitoring of the changes in $\mathrm{PaCO}_{2}$ and FEV1. This loss to follow-up and refusal to undergo additional testing were, unfortunately, out of our control. Second, we did not investigate the potential relevance of $\mathrm{PaO}_{2}$ as a prognostic factor in patients with COPD; this merits further consideration in future studies. Third, the proportion of smokers was high, which may introduce bias. In addition, differences in treatments received among the patients of the two groups may influence the results. Additional studies in larger sample sizes are warranted to address these issues.

In conclusion, increases of age, Charlson Index, chronic hypercapnia and Cor pulmonale, and decreases of FEV1\%, use of medication, BMI and NPPV, were associated with a poor prognosis in patients with COPD. Results can help in correctly evaluating prognosis and improving treatment.

Contributors ZHT and HY conceived and planned the study. PCX, EMZ and WAG extracted patients' data. YWS analysed the data. SZ contributed to the writing of the manuscript. All the authors read and approved the final manuscript.

Funding This study was supported by the Beijing High-grade Talents Health Technology Fund (2011206).

Competing interests None declared.

Patient consent Obtained.

Ethics approval Ethics Committee of Beijing Chao-Yang Hospital and Shou-Gang Hospital.

Provenance and peer review Not commissioned; externally peer reviewed.

Data sharing statement No additional data are available.

Open Access This is an Open Access article distributed in accordance with the Creative Commons Attribution Non Commercial (CC BY-NC 4.0) license, which permits others to distribute, remix, adapt, build upon this work noncommercially, and license their derivative works on different terms, provided the original work is properly cited and the use is non-commercial. See: http:// creativecommons.org/licenses/by-nc/4.0/

\section{REFERENCES}

1. [No authors listed]. Standards for the diagnosis and care of patients with chronic obstructive pulmonary disease. American Thoracic Society. Am J Respir Crit Care Med 1995;152:S77-121.

2. van den Bemt $L$, Schermer TR. Multicomponent staging indices for chronic obstructive pulmonary disease in daily patient care: what's the yield? Int J Clin Pract 2010;64:1475-9.

3. Han MK, Agusti A, Calverley PM, et al. Chronic obstructive pulmonary disease phenotypes: the future of COPD. Am J Respir Crit Care Med 2010;182:598-604.

4. Costello R, Deegan P, Fitzpatrick M, et al. Reversible hypercapnia in chronic obstructive pulmonary disease: a distinct pattern of respiratory failure with a favorable prognosis. $\mathrm{Am} \mathrm{J} \mathrm{Med}$ 1997;102:239-44.

5. Berger KI, Norman RG, Ayappa I, et al. Potential mechanism for transition between acute hypercapnia during sleep to chronic hypercapnia during wakefulness in obstructive sleep apnea. Adv Exp Med Biol 2008;605:431-6.

6. Aida A, Miyamoto K, Nishimura M, et al. Prognostic value of hypercapnia in patients with chronic respiratory failure during long-term oxygen therapy. Am J Respir Crit Care Med 1998;158:188-93.

7. Saryal S, Celik G, Karabiyikoglu G. Distinctive features and long-term survival of reversible and chronic hypercapnic patients with COPD. Monaldi Arch Chest Dis 1999;54:212-16.

8. Nizet TA, van den Elshout FJ, Heijdra YF, et al. Survival of chronic hypercapnic COPD patients is predicted by smoking habits, comorbidity, and hypoxemia. Chest 2005;127:1904-10.

9. Budweiser S, Hitzl AP, Jörres RA, et al. Health-related quality of life and long-term prognosis in chronic hypercapnic respiratory failure: a prospective survival analysis. Respir Res 2007;8:92.

10. Mokhlesi B, Tulaimat A, Evans AT, et al. Impact of adherence with positive airway pressure therapy on hypercapnia in obstructive sleep apnea. J Clin Sleep Med 2006;2:57-62.

11. Burge S, Wedzicha JA. COPD exacerbations: definitions and classifications. Eur Respir J Suppl 2003;41:46s-53s.

12. Bozkanat E, Tozkoparan E, Baysan O, et al. The significance of elevated brain natriuretic peptide levels in chronic obstructive pulmonary disease. J Int Med Res 2005;33:537-44.

13. Jurcut R, Giusca S, La Gerche A, et al. The echocardiographic assessment of the right ventricle: what to do in 2010? Eur J Echocardiogr 2010;11:81-96.

14. Miniati M, Monti S, Basta G, et al. Soluble receptor for advanced glycation end products in COPD: relationship with emphysema and chronic Cor pulmonale: a case-control study. Respir Res 2011;12:37.

15. Charlson M, Szatrowski TP, Peterson J, et al. Validation of a combined comorbidity index. J Clin Epidemiol 1994;47: 1245-51.

16. Sin DD, Anthonisen NR, Soriano JB, et al. Mortality in COPD: role of comorbidities. Eur Respir J 2006;28:1245-57.

17. [No authors listed]. Continuous or nocturnal oxygen therapy in hypoxemic chronic obstructive lung disease: a clinical trial. Nocturnal Oxygen Therapy Trial Group. Ann Intern Med 1980;93:391-8.

18. Zielinski J. Circumstances of death in chronic obstructive pulmonary disease. Monaldi Arch Chest Dis 1998;53:324-30.

19. Zielinski J, MacNee W, Wedzicha J, et al. Causes of death in patients with COPD and chronic respiratory failure. Monaldi Arch Chest Dis 1997:52:43-7.

20. Connors AF Jr, Dawson NV, Thomas C, et al. Outcomes following acute exacerbation of severe chronic obstructive lung disease. The SUPPORT investigators (Study to Understand Prognoses and Preferences for Outcomes and Risks of Treatments). Am J Respir Crit Care Med 1996;154:959-67.

21. Wouters EF, Groenewegen $\mathrm{KH}$, Dentener MA, et al. Systemic inflammation in chronic obstructive pulmonary disease: the role of exacerbations. Proc Am Thorac Soc 2007:4:626-34.

22. Sin DD, Wu L, Man SF. The relationship between reduced lung function and cardiovascular mortality: a population-based study and a systematic review of the literature. Chest 2005;127:1952-9.

23. Ito $\mathrm{K}$, Barnes PJ. COPD as a disease of accelerated lung aging. Chest 2009;135:173-80.

24. Soriano JB, Visick GT, Muellerova $\mathrm{H}$, et al. Patterns of comorbidities in newly diagnosed COPD and asthma in primary care. Chest 2005;128:2099-107.

25. Mapel DW, Hurley JS, Frost FJ, et al. Health care utilization in chronic obstructive pulmonary disease. A case-control study in a health maintenance organization. Arch Intern Med 2000;160:2653-8. 
26. Komori M, Takada $\mathrm{K}$, Tomizawa $\mathrm{Y}$, et al. Permissive range of hypercapnia for improved peripheral microcirculation and cardiac output in rabbits. Crit Care Med 2007;35:2171-5.

27. Pollock JM, Deibler AR, Whitlow CT, et al. Hypercapnia-induced cerebral hyperperfusion: an underrecognized clinical entity. AJNR Am J Neuroradiol 2009;30:378-85.

28. Weitzenblum E. Chronic Cor pulmonale. Heart 2003;89:225-30.

29. Pauwels RA, Buist AS, Calverley PM, et al. Global strategy for the diagnosis, management, and prevention of chronic obstructive pulmonary disease. NHLBI/WHO Global Initiative for Chronic Obstructive Lung Disease (GOLD) Workshop summary. Am J Respir Crit Care Med 2001;163:1256-76.

30. [No authors listed]. Long term domiciliary oxygen therapy in chronic hypoxic cor pulmonale complicating chronic bronchitis and emphysema. Report of the Medical Research Council Working Party. Lancet 1981;1:681-6.

31. Escarrabill J. [Chronic obstructive pulmonary disease (COPD)]. An Med Interna 2003;20:337-9.

32. Eaton $\mathrm{T}$, Lewis $\mathrm{C}$, Young $\mathrm{P}$, et al. Long-term oxygen therapy improves health-related quality of life. Respir Med 2004;98:285-93.

33. Katsenos S, Constantopoulos SH. Long-term oxygen therapy in COPD: factors affecting and ways of improving patient compliance. Pulm Med 2011;2011:325362.

34. Guell Rous R. Long-term oxygen therapy: are we prescribing appropriately? Int J Chron Obstruct Pulmon Dis 2008;3:231-7.

35. Ward NS, Dushay KM. Clinical concise review: mechanical ventilation of patients with chronic obstructive pulmonary disease. Crit Care Med 2008;36:1614-9. 